\title{
The Association between Menopause Symptoms and Risk of Postmenopausal Breast Cancer among nonusers of Hormone Therapy
}

Tricia Li, Jiali Han, Chunyan He

Cancer Res 2016;76(14 suppl): Abstract nr 1746. DOI: 10.1158/1538-7445.AM2016-1741

\begin{abstract}
Background: Hot flashes are a common symptom among women during their menopause transition, likely due to the changes of endogenous hormone levels. Previous research suggested that hot flashes symptoms were associated with a reduced risk of cardiovascular diseases. Few studies have investigated the association of hot flashes symptoms with the risk of breast cancer. Furthermore, the confounding effect by hormone use has not been sufficiently addressed in previous studies.
\end{abstract}

Methods: We investigated the association between hot flashes symptoms and risk of postmenopausal breast cancer in the Nurses' Health Study (NHS). In order to eliminate the confounding by hormone use, we investigated the association only among women who never used hormone therapy. We calculated relative risk of breast cancer using a multivariate Cox model, adjusting for potential confounders, including age, race, family history of breast cancer, age at menarche, age at menopause, parity, age at first birth, body mass index, smoking, alcohol use, and education. We further investigated the severity of hot flashes symptoms in association with risk of postmenopausal breast cancer.

Results: We documented 1079 incidence of breast cancer during 396,166 person-years of follow-up. Hot flashes symptoms were not associated with risk of postmenopausal breast cancer after adjustment for potential confounders $(\mathrm{RR}=1.02,95 \% \mathrm{CI}, 0.90$ 1.15) among non-users of hormone therapy. Relative risk of breast cancer across symptom severity categories (mild, moderate, severe) were 1.01 (95\% CI, 0.87-1.17), 1.04 (95\% CI, 0.88-1.22), and 1.05 (95\% CI, 0.75-1.46), respectively.

Conclusion: These data suggested that menopausal symptoms were not associated with risk of postmenopausal breast cancer.

\section{Identification of Genes with Age-dependent Expressions in Breast Cancer Patients}

Xiang Gu, LuZhe Sun

Cancer Res 2016;76 (14 Suppl): Abstract nr 1506. DOI: 10.1158/1538-7445.AM2016-1506 Published 15 July 2016

\begin{abstract}
Age is the number one risk factor for breast cancer development. The breast cancer incidence rate increases with age, following beta distribution, which is approximately linear in range from 30 to 70 years old ${ }^{1}$. Transcriptome alterations have been shown to promote tumorigenesis for many types of cancers. Therefore, we hypothesize that the genes with altered expression during aging may promote breast cancer development. Using TCGA data, we extracted whole transcriptome profiling data of matched normal tissues from 82 female patients with age at diagnosis and menopausal status available. The RSEM estimated raw reads counts data were normalized by library sizes, $\log 2$ transformed and further normalized by quantile normalization. Removal of $25 \%$ of genes with lowest mean expressions resulted in subsequent analyses on 15,398 genes. We applied simple linear regression to study the association between gene expression level and age at diagnosis on all the 82 patients, and on the 54 postmenopausal patients to reduce the leverage effect of premenopausal patients with limited age range. The genes with significant correlation are filtered by the criteria: 1) R2 value is among the highest $5 \%, 2$ ) absolute value of slope for age is among the highest $5 \%$ and 3) adjusted $\mathrm{p}$ value for the slope is among the lowest $5 \%$. We identified 210 upregulated and 98 downregulated genes in all patients, as well as 103 upregulated and 164 downregulated genes in postmenopausal patients only. The unions of these genes (258 upregulated and 240 downregulated) are considered as genes affected by age. A combination of methods using moderate hierarchical $t$ test (R/limma) and moderate fitting based on negative binomial distribution (R/DESeq2), with and without Surrogate Variable Analysis for potential confounders (R/SVA), results in identification of 498 upregulated and 256 downregulated genes altered by menopause by comparing postmenopausal to premenopausal patients (FDR $<0.05$ ). Exclusion of these menopause
\end{abstract}


affected genes from those genes affected by age (258 upregulated and 240 downregulated) results in 148 upregulated and 189 downregulated genes during aging. Ingenuity Pathway Analysis shows that these genes are significantly enriched for functions associated with tumorigenesis and functions in cancer. In summary, the transcriptome profiling alterations during aging are associated with development of cancer. Functional analyses of some of the age-associated genes are currently underway.

Funding: CPRIT Research Training Award (RP140105)

\title{
Reference
}

1. Francesco Pompei and Richard Wilson (2001). Age Distribution of Cancer: The incidence Turnover at Old Age. Human and Ecological Risk Assessment: Vol. 7, No. 6, pp. 1619-1650.

\section{Correlation of Expression of ER, PR with the Onset Age, Histopathological Grading, Size of Tumor and Metastatic Behavior of Invasive Duct Cell Carcinoma Breast}

\author{
Int J Med ResRev2016; 4(6): 1024-1029. DOI: 10.17511.ijmrr.2016.i06.27.
}

\begin{abstract}
Carcinoma breast is a heterogeneous malignancy with distinct age of onset pattern. Age specific incidence profile of breast carcinoma shows exponential rise until menopause and then slow after menopause. Largely early onset breast carcinoma represents inherited cause and late onset breast cancer following extended exposure to cancer promoters. Expression of ER shows gradual increase beginning in 3rd decade to a plateau by 6th decade. The present study is aimed at the correlation of expression of ER, PR with the onset of age of breast cancer, histopathological grading, size of tor and metastatic behavior.
\end{abstract}

Material and Method: The study was carried out in 80 cases of invasive duct cell carcinoma. Clinicopathological findings, histopathological grading and immunohistochemistry for ER and PR expression were performed.

Result and Conclusion: Majority cases (85\%) of invasive duct cell carcinoma fall in 31 to 60 years of age group. Most of the patients show more than $2 \mathrm{~cm}$ size (90\%). Majority of the patients fall in histopathological grade II (67.5\%) followed by grade I ( $27.5 \%)$. Lymph node metastasis was seen in 45\% cases in all grades. ER and PR expression show declining trend with age of onset of breast carcinoma. It reflects dysregulated ER and PR expression which contribute to the breast carcinoma etiology. Loss of ER expression result in to distinct age of onset pattern, therefore early onset breast cancer may be benefitted by hormone therapy. Metastatic behavior is independent of ER and PR expression.

\section{Exploring the Role of the Ovarian Stromal Extracellular Matrix in Early Tumor Development}

Jamie T. Goodner-Bingham, Yang Yang-Hartwich

Cancer Res 2016;76(14 Suppl): Abstact nr 923. DOI: 10.1158/1538-7445.AM2016-923

\begin{abstract}
Background: Advancements in ovarian cancer research have shown evidence for extra-ovarian origins of malignant cells. The fallopian tubes (FT) are thought to be a source of the most common and aggressive type of ovarian tumor, high grade serous which are more common in women with increased ovulation. We have previously demonstrated that the ovulation wound site provides an ideal environment for malignant FT cells to implant into the ovary (1). However, while malignant cells implant during the reproductive stage, the highest instance of ovarian cancer occurs in peri- and postmenopausal women. We hypothesize that the ovarian environment provides the necessary factors to keep implanted malignant cells quiescent until peri- and postmenopause when the changes in the ovarian environment trigger proliferation and growth. The extracellular matrix (ECM), secreted by the ovarian stroma, is a major part of this environment which regulates cell-to-cell communication and maintains
\end{abstract}


structural integrity. The intent of this study is to determine the critical role of the pre- and postmenopausal ECM and elucidate the effects of matrix remodelling on malignant cells.

Methods: We generated malignant cells by introducing p53 mutations to healthy FT and ovarian surface epithelial (OSE) cell lines. In vitro, we isolated ECM from premenopausal ovarian stroma. Additionally, we overexpressed matrix metalloproteinase 2/9 (MMP2/9) in stromal cells to induce matrix remodelling, resulting in an ECM resembling postmenopause. These matrices were used to develop a new 3D culture method which allowed us to evaluate the effect menopausal ECM remodelling has on malignant cell pathways.

Results: The 3D ovarian stromal ECM maintained and delayed growth of the co-cultured malignant cells compared to their monolayer counterparts. Postmenopausal remodelling of the ECM, via upregulation of MMPs, triggered proliferation and expansion of malignant cells. We discovered, through pathway screening, that AKT, ERK 1/2, PTEN, WNT and PYK2 were associated with these ECM-related changes in the malignant cells.

Conclusion: These results support the use of a 3D model in culturing pre-tumor cells to create an in vitro model that closely resembles an ovarian environment. We have also shown that the ECM plays a role in maintaining malignant cells by supressing proliferation and growth. Additionally, we have demonstrated that the remodelling of the ECM that occurs with menopauseassociated increase in MMP2/9 expression triggers expansion of malignant cells. It is clear that the ECM plays an important role in the early development of ovarian cancer and that MMP could be a contributing factor to the postmenopausal presentation of these malignancies. Going forward, we plan to further elucidate the specific molecular mechanisms involved in postmenopausal tumor development.

\title{
Comparative Efficacy of Nonhormonal Drugs on Menopausal Hot Flashes
}

Li, L, Xu L, Wu J, Dong L, Zhao S, Zheng Q

Eur J Clin Pharmacol 2016 Sep; 72(9): 1051-8. DOI: 10.1007/500228-016-2090-5. Epub2016 jul 24.

\begin{abstract}
Purpose: The effects of nonhormonal drugs on menopausal hot flashes are still not well quantified. We therefore did a modelbased meta-analysis (MBMA) to quantitate and compare the efficacy features of nonhormonal drugs on menopausal hot flashes.

Methods: Literature was searched in the public databases to extract data of clinical trials on nonhormonal drugs, including selective serotonin reuptake inhibitors (SSRIs) or serotonin norepinephrine reuptake inhibitors (SNRIs), gabapentin, clonidine, and soy isoflavones. Pharmacodynamic models were used for the quantitative analysis of each drug.

Results: Thirty-nine studies were included in the analysis. The results revealed a classic pharmacodynamic maximal effect $\left(\mathrm{E}_{\mathrm{max}}\right)$ model could describe the time course of hot-flash reduction by nonhormonal drugs. After deducting placebo effects, the $\mathrm{E}_{\max }$ of SSRIs/SNRIs, gabapentin, clonidine, and soy isoflavones was $13.9 \%, 14.8 \%, 18.5 \%$, and $25.0 \%$, respectively. The time to achieve half of the maximal effect (ET(50)) of SSRIs/SNRIs, gabapentin, clonidine, and soy isoflavones was $0.18,0,0$, and 11.6 weeks, respectively. The results showed that SSRIs/SNRIs, gabapentin, and clonidine had a rapid onset, which could reach the maximum effect immediately. However, the onset of soy isoflavones was very slow, and a duration of 16.6 weeks was needed to surpass the efficacy of paroxetine (a type of SSRIs).
\end{abstract}

Conclusion: The information provided in this study can be used as valuable supplementary information for treatment guidelines of nonhormonal drugs on menopausal hot flashes.

\section{Method of Treating Thermoregulatory Dysfunction with Paroxetine}

PAT Richards - US Patent 9,393,237, 2016

\section{ABSTRACT}

The present invention relates to a method for treating a patient suffering from a thermoregulatory dysfunction, especially hot flashes and flushes associated with hormonal changes due to naturally occurring menopause (whether male or female) or due 
to chemically or surgically induced menopause. The method is also applicable to treating the hot flashes, hot flushes, or night sweats associated with disease states that disrupt normal hormonal regulation of body temperature. The invention further relates to use of paraxetine or a salt thereof.

\title{
A Selective Estrogen Receptor Beta Agonist for the Treatment of Hot Flushes: Phase 2 Clinical Trial
}

Mary A. Tagliaferri, Margit C. Tagliaferri, Jennifer M. Creasman, William D. Koltun

The Journal of Alternative and Complementary Medicine September 2016;22(9): 722-728. doi:10.1089/acm.2015.0021.

\begin{abstract}
Objective: The aim of this study was to evaluate the safety, tolerability, and efficacy of a selective estrogen receptor beta (ERD) agonist, Dr. Tagliaferri's Menopause Formula (MF102), to treat the symptoms of menopause.

Methods: An open-label trial of MF102 taken for 12 weeks by 30 postmenopausal women aged 40 to 65 years, who experienced a minimum of five moderate to severe hot flushes per day. The primary efficacy outcome was a change in the frequency of moderate to severe hot flushes from baseline to week 12. A change in the frequency of hot flushes that woke participants from their sleep from baseline to 12 weeks was a secondary endpoint. Lipid profile and endometrial thickness were also evaluated.

Results: Thirty postmenopausal women with an average of nine moderate to severe hot flushes per day were treated with MF102 4g/day; 27 participants completed the study. The median percent reduction in moderate to severe hot flushes was $71 \%$ $(\mathrm{p}<0.001)$. The median percent reduction in hot flushes that woke participants from their sleep was $54 \%$ ( $p<0.001)$. Low-density lipoprotein (LDL-C) and total cholesterol both declined significantly from baseline. There were no serious adverse events, reports of abnormal uterine bleeding, or significant changes in double-wall endometrial thickness.
\end{abstract}

Conclusion: Treatment with MF102 resulted in a marked decrease in the frequency of moderate to severe hot flushes, was welltolerated, and demonstrated no safety concerns.

\section{Endometrial Carcinoma in a Young Female: Report of Two Cases}

Osman Ali, Ibrahim Abdillahi, Majdouline Boujoual, Jaouad Kouach, Moussaoui Idrissi, Mohammed Dehayni International Journal of Innovation and Applied Studies 16.3 (Jun 2016): 570-572.

\begin{abstract}
Endometrial cancer (EC) is the most common gynecologic malignancy in developed countries and affects predominantly postmenopausal women. It is estimated, however, that 15 to $25 \%$ of women will be diagnosed before menopause. Total abdominal hysterectomy, bilateral salpingo-oophorectomy (BSO), and surgical staging are usually performed in the treatment of endometrial cancer. Further treatment is tailored according to the presence or absence of various risk factors. That conservative treatment of early-stage endometrial adenocarcinoma in young women wishing to preserve fertility should be considered in carefully selected cases. Assisted reproductive technologies may be helpful for immediate achievement of pregnancy in such patients. We report managements of two young patient of endometrial carcinoma.
\end{abstract}

